\title{
An Detailed review on Applications of Smart Materials in Structural Engineering
}

\author{
Udula Mangalika \\ Department of Mechanical Engineering, University of Peradeniya, Sri Lanka. \\ ludula@aol.com
}

\begin{abstract}
ArticleInfo
Journal of Machine and Computing (http://anapub.co.ke/journals/jmc/jmc.html)

Doi : https://doi.org/10.53759/7669/jmc202101007

Received 05 December 2020; Revised form 25 January 2021; Accepted 25 March 2021; Available online 05 April 2021.

(C)2021 The Authors. Published by AnaPub Publications.

This is an open access article under the CC BY-NC-ND license. (http://creativecommons.org/licenses/by-nc-nd/4.0/)

Abstract - Smart structural materials are a fundamental aspect that requires critical analysis in in structural engineering. This research contribution considers two vital crystal structures: Martensite and Austenite with their characteristic variations under different temperatures. From this research, it is seen the smart materials presents two vital features, which are distinguished from typical steels. The first one is the memory shape and the second one is super-elasticity. All of these properties can suit various applications in structural engineering e.g. dual actuators, self-rehabilitation and pre-stress bars. This research is purposed at analyzing the applications of smart materials in the field of structural engineering through the focus of relevant literature reviews, typical collection of data and major mechanic elements of smart materials. In axial tension evaluation, the curve for force and extension and the strain and stress curves of super-elasticity and Shape Memory Alloy (SMA) materials have been considered. The beam experiment with the superelasticity materials considered as the bars for reinforcement has been considered as well. This contribution provides the initial step of evaluation of the wide-range application of smart materials in the field of structural engineering.
\end{abstract}

Keyword - Smart Materials (SMs), Martensite, Austenite, Super-Elasticity (SE), Shape Memory Alloy (SMA).

\section{INTRODUCTION}

Considering the present state and development of technology for engineered materials, numerous materials have found their application in structural engineering and in handling the present deteriorating condition of infrastructure. Many novel cost-efficient and high-quality materials have been utilized in the field of structural engineering. Smart Materials (SMs) are one of them. From the past evaluations of Nickel and Titanium SM done, it was noted that the technological application could be applied in various fields; e.g. biomedical, mechanical and aerospace engineering. In the modern age, engineers and researchers are concentrating on SMs for their critical features and performances in structural engineering [1]. SMs are available in two stages at various temperatures. The first stage is Austenite that is typically present at extremely high temperatures and the second one is Martensite that typically exists in lower temperatures. Whenever the external temperatures or the status of stress is twisted, the dual stages will change to another stage, and this depends on the changes appearing.

SMs display many critical characteristics during their processes of change between the dual stages. One of the characteristics is effect of the Shape Memory Alloy (SMA), Super-Elasticity (SE) and the two-path effect of memory. Certainly, the most clear indicates the capacity of SMA, which is displayed in commercials where sunglasses can be changed around the user's fingers. When an individual releases them, sunglasses tend to snap to their appropriate shapes. This is confirmed as SE and it normally the feature of SMA, which permits them to behave in this manner. As these wires tend to bend, the enhancing stress stimulates the wires to change from being Austenite to being Martensite.

Nonetheless, whenever these wires have been released, stress diminishes to zero and these wires transform to the Austenite stage and its typical shape. The aspect of SMs in structural engineering is rapidly transforming considering the present advancement of technological application, which includes shape-processing, actuators, sensors, and engineered materials among others. The aspect of smartness denotes self-sensing, self-adaptability, multiple functionality and memory of the structures and materials. All of these features provide wide-range potential application of structures and materials in aerospace processing, structural systems, and biomechanical engineering. Self-adaptability feature of SM is advantageous and applies embedded adaptation of SM such as SMA [2]. Through the twist of these features, SM can potentially identify cracks and faults and are thus significant as a tool for diagnosis. This feature can be used to effectively activate SM that has been embedded in the host materials in an effective manner to deal with these faults. This aspect is considered as selfrepairing impact.

SMA represents a critical category of adaptive materials, which can change thermal power to mechanical operation directly. Whenever this is, effectively mixed, different alloys display this aspect through repeated treatment of heat. The effect of shape memory in different alloys is properly documented since the early $19^{\text {th }}$ century, which displays the 
commercial products being considered in the aerospace and mechanical industries. Ni-Ti is considered as the mostly utilized SMA [3]. Whenever SMA has been subjected to plastic deformation at a lower temperature, it normally goes through the mechanical twining procedure whereby every adjacent atom layer moving by a single lattice parameter.

Nonetheless, when materials have been heated over Austenite finishing temperature, it tend to undergo the stage change, and recovering wide-range mechanically based restoring and deformation to its original shape of memory and its specimen. This effect is considered as the one-path memory influence. Based on the special heat treatment procedure, the two-path memory influence can be accomplished. The two-path memory alloy can potentially undergo opposite but significant deformation upon cooling and heating and can potentially act as a two-path actuator. Heating SMA amounts to a single memorized shape whereas cooling amounts to a second varied shape [4]. Utilizing this two-path impact allows SMA to effectively shrink or expand so that is can effectively remove and apply stress in structures accordingly and this amounts to smart engineering structures.

The staged transformation temperature is dependent on not only the composition of materials, but also the state of stress. Whenever stress transforms, the phased transformation temperature can also transform. This implies that the stage transformation can be activated without real transition in temperature, but based on stress transformation, as in the instance showcase in the sunglass example mentioned earlier. This feature gives a fundamental application of individual repair. Whenever concrete structures begin their cracking process, the enhanced stress in SMA can influence the transformation phase that produced the stress of compression in the relevant structures hence limiting the cracking process.

Another significant feature of SMA is that temperatures tend to increase hence amounting to the shrinkage during the process of change. This form of shrinkage can be used to effectively balance thermal expansion. Thus, SMA can be considered as a promising material that handles the temperatures that have been induced by cracking and stress issues. Differential settlement between the pavements and bridges affects the uneven joints and bumps at ends of the bridge. Whenever motors, mostly heavier trucks leave or approach the bridge, the bumps influence the significant impact load to pavements and bridges. It is considered that the uneven joints might affect the deterioration of pavements and bridges, cause accidents and negatively affect automobiles. The damages also incorporate the separation of pavement toppings from the bases, spalling of joints, cracking fatigue and damages of bridges. This same issue has been connected to the uneven settlements between the approach span and bridge piers. Whenever the differential settlements happen, it is in bridge drivability affected, however, more internal forces can also be formed for the structures [5].

Joint maintenance is a significant issue and highway officials and engineers incur the costs. Earlier, when mitigating this issue, engineers concentrated on developing a foundational structure. Nonetheless, they have not managed to resolve these issues connected to the unevenness of joints and wide-range varied settlements. The changes in temperature and the time-dependent aspect affect i.e. shrinkage and creeps; also affect the internal restraint forces for the indeterminate structures. The forces with other relevant load impacts affect the cracking of the present pavements and structures, which also enhance the expenses of novel constructions designated to mitigate these issues and effects ice increment of sectional dimension.

Another key issue is the performance of bridge bearings. Factors such as malfunctioned bearing due to deterioration of materials, dirt clogging and other relevant factors influence stress of the bearing segments. Resultantly, this impacts change in redistribution of force and therefore makes the bridge to act differently from the projected designs. This issue is a common factor in the failure of bridges and it maintenance necessities. Identifying a manner to effectively transform forces in bearing to alleviate the issues discussed above is considerably. The two-path memory influence of SMA can be utilized to make the actuators of SMA, which can fail or rise to transform the heights. SMA can be utilized to process the smart strands. After the mechanical deformation of the strands and embedment have been done in concrete, self-repairing and pre-stressing impacts can be considered as required during the structural life cycle. Smart strand are normally actuator, which can normally activated through internal stress and heating transformations [6].

This section (Section I) has introduced the paper by evaluating SM as one of the cost-efficient and high-quality materials used in the field of engineering. Considering the status of developments in engineered materials and technology, it is considered the SMs have widely been applied in the field of structural engineering. In this research, SMs is available in two stages at various temperatures. The first stage is Austenite that is typically present at extremely high temperatures and the second one is Martensite In that regard, the remaining sections of the paper present a critical analysis of Smart Materials (SMs) in structural engineering. The rest of the paper is organized as follows: Section II presents the background analysis of the research. Section III focusses on literature review of the topic. Section IV is the methodology part. Section $\mathrm{V}$ presents experimental analysis of the research. Section VI is the discussion of results. Finally, Section VII concludes the paper and presents future directions.

\section{BACKGROUND ANALYSIS}

This research contribution concentrates on the application of Smart Materials (SMs) in structural engineering through the gathering and reviewing of the relevant literature in Section III. This will allow us to gather basic knowledge concerning the smart materials before focusing on the major structural elements of SMs. The property of SMs tests have been based on the beam experiments, which are done to retrieve the data about the parameters of the materials utilized in this contribution and to effectively verify the wide-range application of SMs in structural engineering i.e. custom rehabilitations of cracked concrete segments. 
In this research, the findings will act as a foundation, which will project future research in this field. This paper projects the analysis on pre-stress, individual repair capacities and two-path memory trainings that will definitely facilitate the developments of smart bridge scheme as seen in Fig 1.

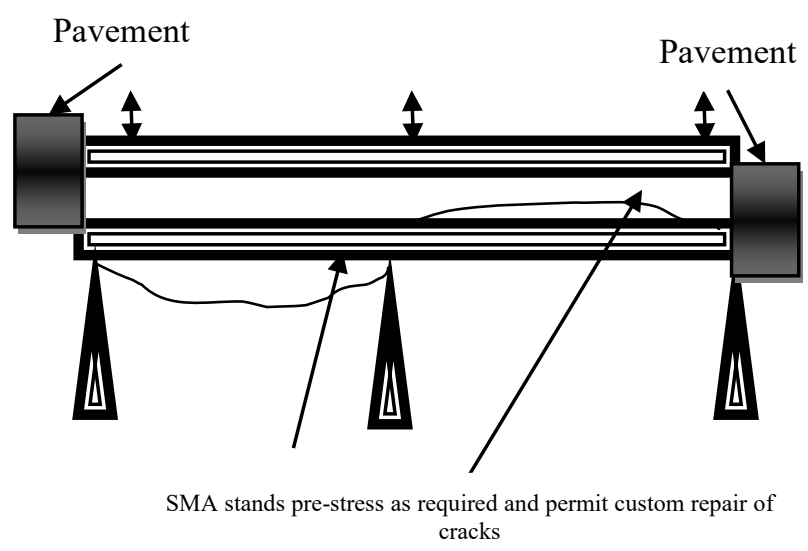

Fig 1: Smart Bridge Scheme

This research contribution has considered certain tasks: The first task considered in this research is the analysis of the relevant literatures to gather the essential data considering the present SMs products. Various SMs as considered, contrasted in this analysis, and staged based on their transformation temperatures, and material characteristics. The second task is material testing where the stress/strain and force/extension curves on the shape memory materials have been drawn. The third task focuses on the beam experiment where the points of concrete beam with super-elasticity SMs have been considered. The fourth task is the cracking of custom rehabilitation of the concrete beam with super-elasticity SMs.

From this research, the application of the smart strands and bearing can be considered in the development of the smart bridge as indicated in Fig 1. Smart bearing will transform the heights based on effects of shape memory of SMA. The height changes will rectify the unevenness issue, which considers the precise unevenness issue, which incorporate the internal forces that has been induced from varied settlements, pre-stressing relaxation, concrete shrinkage and the transition in temperatures as evaluated earlier on.

Whenever required, the pre-stress force can be changed to handle the cracking problem in both the negative and positive moment segments. With the interlinked applications of smart bearing and strands, the bridge can change its inner force distribution and mobilization of elements to effectively adapt to different ecological conditions and loads such as the ones induced by differential settlements, temperature impact, and time dependent impacts. The main purpose of this research contribution is to evaluate data on SMs, which potential allows the structuring of smart bridge schemes. Section III below discusses the relevant literatures of SMs and SMA, which also incorporates the evaluations and measurements of transformational temperatures.

\section{LITERATURE REVIEW}

M. Akita in [7] confirm that a system with the capacity to respond and sense in the pre-determined way and in a managed form with outside stimuli can effectively revert to its initial condition when the outer stimuli is eliminated. Smart structural schemes have been designed in a manner that can effectively detect the damages and might potentially control the responses from outer disturbances. In cases of earthquakes, these damages are normally affected by multi-storey structures, and are typically overturning, sliding, bending and shearing damages. These damages tend to occur in concrete shear walls.

J. Clark in [8] agrees that in the designing of smart material, various forms of materials are considered. These materials are capable of sensing and responding to various conditions having a single or multiple properties, which can incredibly transform in a controlled manner in an outer stimuli e.g. temperature, pressure, electric and moisture. In this case, Shape Memory Alloy (SMA) is considered. Others include polymers sensitive to temperature and piezoelectric. Overall, SMs are capable of transforming their color, size and shape based on external stimuli.

S. Kim and H. Sohn in [9] argue that materials have superior features e.g. plastics, composites, ceramics, alloys and advance metals, which cover the fundamental materials for advance processing i.e. Electrorheological Fluid (EF) for multimaterials 3D printings and haptic feedbacks. W. Choi in [10] assessed the environmentally sensitive SMs, which have been structured to be sensitive to the environment and these can be derived from petroleum, such as the waste materials, mycelium-centered materials, bioplastics and physical fiber composite.

J. Zhang and C. McInnes in [11] have focused on the smart structures that are capable of holding all the essential parameters fundamental to be a smart structure. To formulate adaptive structural scheme to ecological transformations, we have to be capable to determine these transformations via sensor technologies based on the capacity of materials to change the mechanical motion into electric signal with basis on the correctness degree. Sensors can be piezo-electric accelerometer 
or the foil form of strain transducer and the optical fiber sensors. Whenever handling the signal generating sensors, it is fundamental to clean and isolate signals for precise signal interpretation. Traditionally, piezo-electric materials are utilized in the strain sensors, receptors and emitters of pressure transducer, actuators and stress waves.

S. Klinkel, D. Legner and W. Wagner in [12] argue that piezo-electric structures and materials have been utilized extensively in vibration damper and in the control of moving or stationary structure e.g. the airplane blade. Whenever applying tensile or compressive stress in the polarization direction of materials, it will produce electric fields with opposite voltage polarities and therefore materials will contract and expand based on the applicable fields.

T. Breczko, V. Barkaline and Y. Douhaya in [13] classify SMA in the category of metallic alloys, which can be reformed or deformed to their initial shapes whenever heated above their transformational temperatures. They can potentially recover their original shapes after the process of deformation is complete; for instance, NITINOL, which are the first, structured SMA and an alloy of Titanium and Nickel. Nowadays, SMA is generated based on the combinations of Zinc and Copper. SMA are utilized in joints, and screws for the establishment of underwater (this will help to eliminate the underwater welding). The materials can be able to undergo significant strain, which is about $10 \%$ without any significant deformation of materials left behind. The major element of SMA is its effect of the shape memory whenever materials are in martensite manner i.e. brittle and hard solid solutions of iron and carbon, which are fundamental hardening constituents.

D. Vojtěch and A. Michalcová in [14] evaluated the NITINOL temperatures of SMA. In the confirmation, various forms of transformation temperatures were considered. Martensite starting temperatures were considered as the temperatures at which transformation from the martensite states and austenite states start the process of cooling. The second form is martensite finish temperatures represent the temperatures at which transformations from austenite states to the martensite states start on heating. The third form is austenite-finishing temperatures that signify the temperature at which the transformations from the martenite states to austenite states complete on heating.

S. Dokhov in [15] evaluated the measurement of transformational temperatures. This is meant to determine the change temperatures of NITINNOL SMA, differential scan calorimetry is considered through the evaluation of energy flow between NITINOL and the ecosystem. This is considered as a fundamental function of temperatures. To evaluate the shape recovery aspect of specimen as the temperature function, the bend and free recoveries tests can be conducted. Transformation temperatures for actuator and fastener application are evaluated by considering the constant load dilatometry procedure,

An analytical research to assess the efficiency of SMA and piezoelectric in structural engineering is missing and needs critical analyzing namely seismic isolations of concrete shear wall. Utilizing the computer modeled ABAQUS, two forms of walls: ordinary and another connected with shear walls. Moreover, dynamic response in this instance of earthquakes can be considered. Other than steel bars, super elastic SMAs have affected remarkable reduction in residual and reduction displacement. Various SMAs combination with steel reinforcement has been modeled in various structures. The section below discussed the methodology considered for this research.

\section{METHODOLOGY}

To underpin this research, a literature review of the relevant scholastic texts was evaluated to evaluate the smart materials that can potentially be used in structural engineering. Relative novel materials i.e. smart materials have been utilized widely over the past few decades in biomedical, mechanical and aerospace applications.

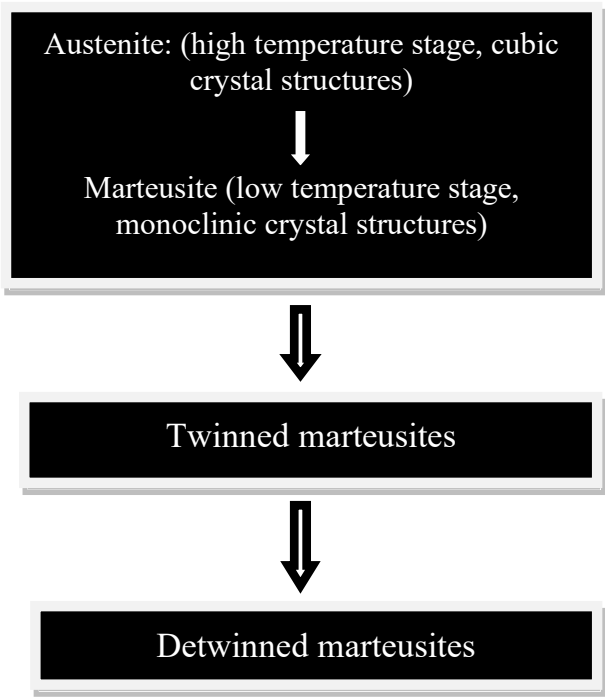

Fig 2: Various stages of SMs 
Based on the findings of the research, some application has been evaluated for structural engineering lab experiments and fieldwork practices. The first recorded observations of SMs transformations were structured in 1930s in gold cadmium. In the late 1930s, staged transformation was witnessed in copper-zinc. In 1960s, coworkers and Beehler founded the attendant and transformation SMA impact in Nickel Titanium at the lab in Naval ordinance.

\section{A. Transformation Method of SMs}

SMs exist as two stable stages under various temperatures: Martensite i.e. the low temperatures stage and Austenite i.e. the high temperature stage. Moreover, there are two various types of Martensite materials: detwinned and twinned as indicated in Fig 2 below. At a particular temperature, SMs will stay at their designed thermodynamically stable stage.

Nonetheless, whenever these temperatures change, the transformations between the two different stages occur. Because of this transformation method, SMs have a critical feature considered e.g. SMs effect and the super-elasticity impact, which has a significant potential application in structural engineering. SMs are one of the fundamental features considered in structural engineering. Whenever SMs in twinned Martensite stage undergo exterior stress, it can potentially change during this process of transformation. When detwinned martensite material increase in temperature, the detwinned martensite change to austenite as seen in phase 2 of Fig 3 below. According to phase 3, Austenite will shift to the twinned martensite whenever temperatures drop. There are four fundamental feature temperatures between phase 2 and phase 3 considering the ascending temperature form: Austenite finish temperature, Austenite start temperatures, Martensitic start temperatures, and Martensite finish temperatures.

Twinned M

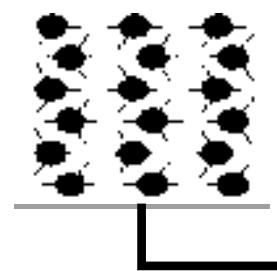

Austenite

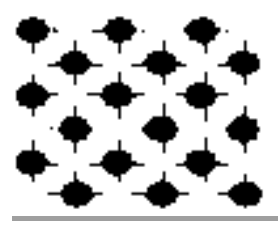

Detwinned M

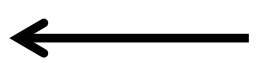

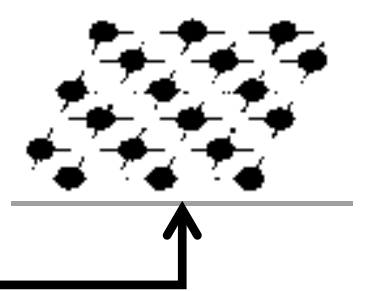

Fig 3: Changes between various phases

In phase 1, 2 and 3, the SMs will undergo exterior stress transformation, increase in temperature and decrease in temperature. Finally, it can potentially shift to its initial twinned condition. Phase 3 constraints, the recovery phase from Austenite to twinned martensite will potentially provide significant stress. This certain characteristic can be utilized in various ways in structural engineering and civil engineering application.

Super-elasticity is the second most significant characteristic of SMs. When Austenite-finish temperatures are significantly low, super-elasticity materials will be considered as Austenite temperatures i.e. below room temperatures. In the Ausstenite stage range, super-elasticity materials can change into significantly detwinned martensite under pressure. Nonetheless, detwinned martensite materials will shift to Austenite whenever exterior pressure has been released, as shown in Fig 4 below.

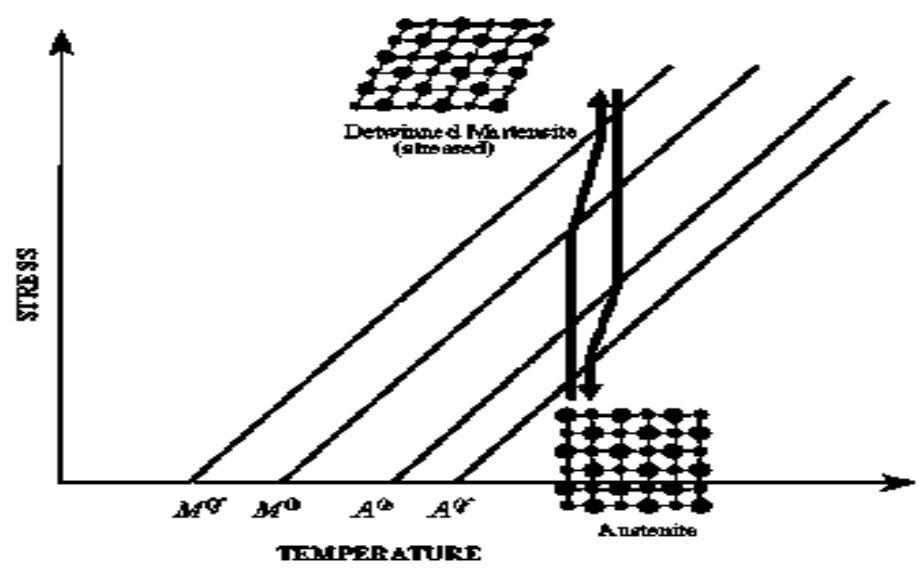

Fig 4: Stress-based transformation of Austenite materials 
Fig 5 below shows the stress and strain connection of martensite and austenite. Based on the stress and strain perspective, SMs operate differently in Austenite and Martensite stages

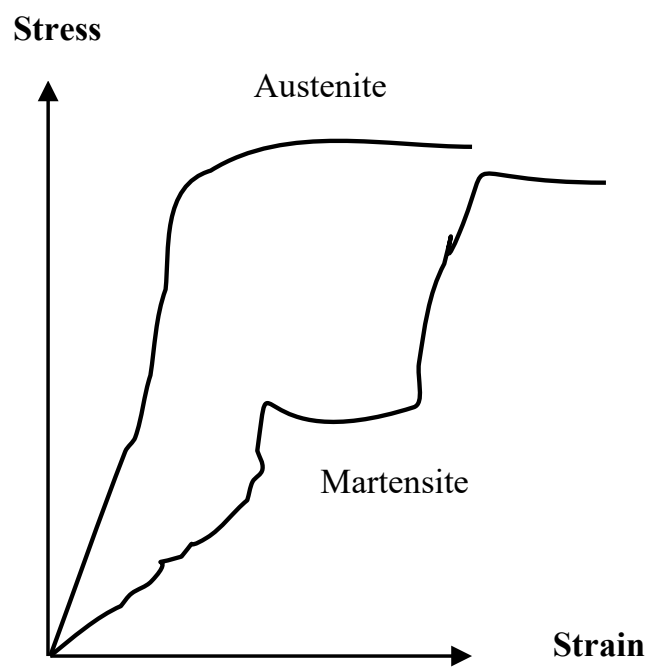

Fig 5: Stress and strain relation of martensite and austenite (sketch)

According to Fig 5, a normal stress and stress curve of SMs represents two stages. The top-most plateau signifies the transformation of Martensite from Austenite subjected under pressure whereas low plateau signifies the reverse procedure. with the release of stress. This characterisitc can be utilized to potentially rehabilitate cracked concrete whenever superelasticity SMs are utilized as reinforcement bars. The super-elasticity materials are known for some significant characteristics such as hysterical damping, significant reliable energy dissipation capability via repeatable stage change, smart fatigue features and smart resistace to potential corrosion.

\section{B. Model Analysis of SMs}

After mecanical deformation process has been completeted, SMs can hold onto their initial configurations through the process of heating alloy above the temperature of transformation. Using this process, the recoverable strains, which can be more than $8 \%$ can be retrieved. To effectively comprehend the mechanical element of SMs, constitutive schemes for SMs should be established. These schemes of SMs have been a prevailing concern for SMs. Considering a short review of potential models, ferro-electric models have been seen to focus significantly on thermodynamics, free energy in ferromagnetics, ferro-electrics and statistical physics.

Researchers in [16] have projected a scheme based on the extension of constitutive stage for SMs, which makes it easier to comprehend the changes of the connected behavior of materials. Nonetheless, it is potentially possible to utilize the schemes in assessing the features of microstructures in different procedure whereas challenging to utilize engineeering design applications. Researchers in [17] have also evaluated a scheme of free energy that assumed an element of absolute temperature and high-order stain energy. Numerical findings indicate that the scheme is significant in the process of simulating the transformations of Martensite. Nonetheless, the aspects of computation prohibit it for application in particar structural engineering application.

Researchers in [18] have evaluated the plasticity scheme, which is a model meant to define the constrututive connection of SMs. The model was effectively modified to signify super-elasticity and SMA effect of SMs. The researcers introduced a scheme of single crystal for SMs using experiments. The scheme accounts for significant range of physical occurrences compared to analogue plastic stages based on the dependencies on the time history of streain and stage fractions. The model also operatures for single crystal SMs in smooth transformauton procedure from elastic to plastic modes. The researchers proposed the Hysteresis model that is 1D constitutive scheme that is critically based on the hystereris model of the damped materials. The model is analogous in inelastic formulation for visco-elesticity and creep that contain the back stress.

As such, the model is capable of represeting both elastic and inelastic condition and the variations between loading or unloading in SMs. It can project both the Martensite and Austensite twinning hysteresis for SMs. Non-isothermal models was proposed in the early 1990s. This model incorporates the relationships between the full transformation aspect and the arrested unloading and loading. The scheme also puts into consideration the change to adiabatic from isothermal loading via the loadingin energy convective ecosystems. Due to the ideology that the scheme has one variant in any stage, it can not capture particular phenomena in twinning that are normally connected to SMA effect. 


\section{Application of SMs}

SMs have widely been applied in various engineering fields such as in shape control, coupling, composite, actuators, shock absorpotion, automatic on and off switches and vibration dumping segments. Under the quasi-static condition, SMs have been applied in load bearings actuators available in various complex structures e.g. motor blades, aircraft wings and submersibles.

In the sector of biomedical, wide-range investigators are undertaking analysis on the underwater motors, which can potentially share hyrodynamic efficacy and agility of aquatic living organisms. SMs are known for the capacity to transform there interior forces. Based on this characteristic, adjustments are posible and might potentially lead to particular dynamic and static features of the composite material structure, which can be set precisely. The structures to be considered in this case include shape and deflection effects. Moreover, vibration modes, natural frequencym damping elements, and amplitudes of stressed vibrations can be considered as desired figures. The section below discussed the experimental analysis, which considers the material features and the material tests.

\section{EXPERIMENTAL ANALYSIS}

\section{A. Basic Material Features}

After contrasting the varuous SMs based on staged transformation temperatures, price and material features, critical metal corporations were selected as the materials to consider in this analysis: NDC and Ni-Ti alloys (see Table 1).

Table 1: SMs for this research

\begin{tabular}{lll}
\hline & NDC & Special Metal \\
\hline Shape Memory & SE-508 & \#C\&7078-21 \\
& & \#C\&7077 \\
Superelasticity & SM-495 & \\
\hline
\end{tabular}

SMs have some major mechanical features generated by companies as indicated in Table 2, Table 3 and Table 4 . The Ni-Ti alloy has higher elongation feature, low Young's modulus, higher ultimate tensile strength contrasted to normal steels.

Table 2: Mechanical features of NDC SE-508 Smart Materials

\begin{tabular}{llll}
\hline $\begin{array}{l}\text { Young's } \\
\text { modulus }\end{array}$ & $\begin{array}{l}\text { Ultimate } \\
\text { tensile } \\
\text { strengths }\end{array}$ & $\begin{array}{l}\text { Overall } \\
\text { elongation }\end{array}$ & $\begin{array}{l}\text { Temperature } \\
\text { transformation }\end{array}$ \\
\hline $\mathbf{6 . 1 1}$ by & 160.200 by & $10 \%$ & $\begin{array}{l}5.18 \text { degrees } \\
\text { Celsius }\end{array}$ \\
\hline $\mathbf{1 , 0 0 0 , 0 0 0}$ & 1000 & & \\
\hline
\end{tabular}

Table 3: Mechanical features of NDC SM-495 Smart Materials

\begin{tabular}{lllll}
\hline $\begin{array}{l}\text { Young's } \\
\text { modulus }\end{array}$ & $\begin{array}{l}\text { Ultimate } \\
\text { tensile } \\
\text { strengths }\end{array}$ & $\begin{array}{l}\text { Overall } \\
\text { elongation }\end{array}$ & $\begin{array}{l}\text { Temperature } \\
\text { transformation }\end{array}$ \\
\hline $\begin{array}{l}\mathbf{4 . 6} \text { by } \\
\mathbf{1 0 0 0 0 0 0}\end{array}$ & 160 by 1000 & $10 \%$ & 60 degree \\
\hline
\end{tabular}

Table 4: Mechanical features of special metal super elasticity material

\begin{tabular}{|c|c|c|c|}
\hline $\begin{array}{l}\text { Young's } \\
\text { modulus }\end{array}$ & $\begin{array}{l}\text { Ultimate } \\
\text { tensile } \\
\text { strengths }\end{array}$ & $\begin{array}{l}\text { Overall } \\
\text { elongation }\end{array}$ & $\begin{array}{l}\text { Temperature } \\
\text { transformation }\end{array}$ \\
\hline $\begin{array}{l}12 \text { by } \\
1,000,000\end{array}$ & $\begin{array}{l}100.200 \text { by } \\
1000\end{array}$ & $\begin{array}{l}20 \% \\
40 \%\end{array}$ & $\begin{array}{l}94 \text { degrees } \\
\text { Celsius }\end{array}$ \\
\hline
\end{tabular}




\section{B. Material Testing}

The experimental analysis considered in this subsection is the stress-strain curves, which have been obtained and SMA impact verified. MTS framework Q-test VII and its connected software test works have been utilized in material testing. The model can do various materials testing incorporating axial tensile test that is utilized to evaluate the extension of particular segments of the sample. Software read the extension and loading value directly. During the process of testing, crosshead velocity was set at about 0.2 in per minutes, where the highest load limit was considered $10000 \mathrm{lb}$, while the highest extension limit was considered 0.41 inches. Specified sample length of the sample segment for evaluating the extension is 2 inches.

Fig 6 below represents the connection between the parameters considered and the machines included in the experiments. From Fig 6, RCLM (viewed as manual) is the control and recording of load machine whereas PC signifies custom computer. RCLM governed and retrieved readings are from loading machines. Cracking, displacement and strain can be determined using information acquisition model and there communicated to the PC system. Because RCLM model and information acquisition model have not incorporated in general, force readings from RCLM and subsequent variables from information acquisition scheme are not synchronous and manual tasks required in the analysis of data.

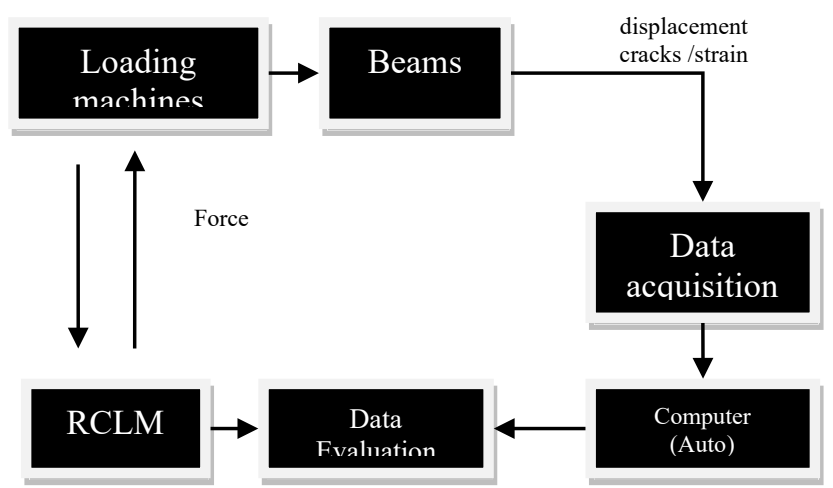

Fig 6: Relationship between machines and parameters of the beam experiment

The loading and reading system for beam experiments is considered based on four-point loading. Middle two supports act as the points of loading whereas the two ends separated from the beam bottom when force is applied upwards. The top part of the beam, two blocks are able to shift upwards and downwards, and operate as two constraints. Moreover, the displacement transducers and the cracked transducers on the beam top are considered as shown in Fig 7.

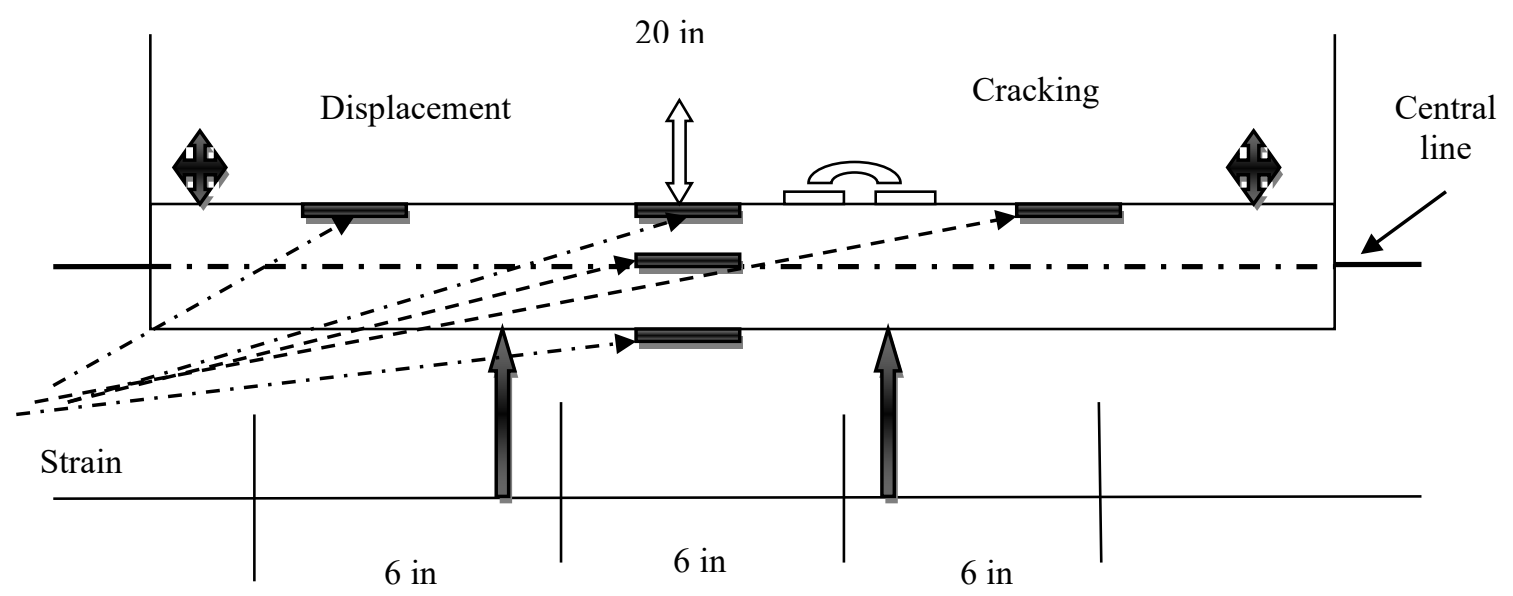

Fig 7: Evaluation model of the beam experiment

Based on the material parameters and geometric parameters in Tables 1, 2, and 3, theoretical cracking moments, strain and ultimate moments at various points on the concrete beam surface can be evaluated following the length of compression that has been obtained from concrete evaluations. Based on this data, unloading and loading processes can be established before the beam experiments. For every beam, $3 \sim 4$ loading and unloading circle have been considered to observe the cracking moments, the transformation of cracking width and other performance measures of the beam. 
In the initial loading circle, the exterior pressure was increasingly applied until the first crack of concrete happened. For was then minimized to 0 . In the $2^{\text {nd }}$ and $3^{\text {rd }}$ loading circles, exterior force was considered to increase the present cracking and then minimized to zero. In the $4^{\text {th }}$ loading, exterior force was enhanced to crush beams. In the initial testing process, width of cracking was considered manually at particular loadings, whereas displacement of midspan, crack transducer, and strains at various points were considered in the entire material testing process.

\section{DISCUSSION OF RESULTS}

Fig 8 represents the load and extension curve and Fig 9 represents the stress-strain curve of NDC super-elasticity materials i.e. SE-508 wires that have a diameter of 0.187. In Fig 9, there are yields of NDC stress super-elastic materials of about $80 \mathrm{~K}$ psi that is typically more than the value of $65 \mathrm{~K}$ psi provided by the engineering firm. From the set of data, Young's modulus can be evaluated as 18.54 by 106 psi that is more than 6.11 by 106 psi given by the firm.

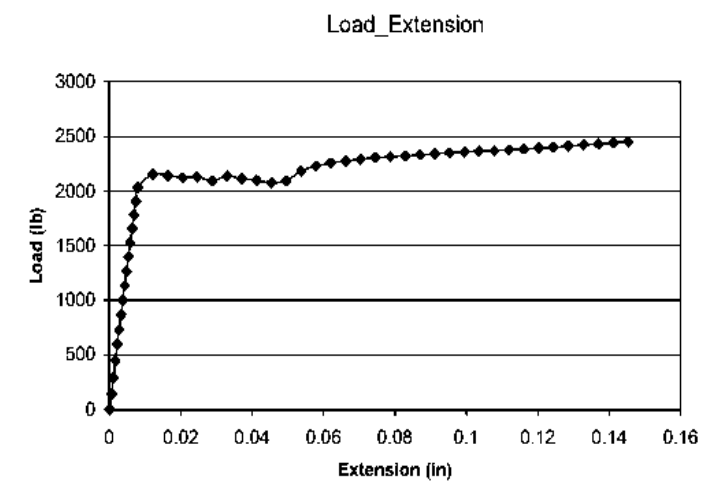

Fig 8: Load and extension curve for NDC super-elasticity materials' width $=0.187$.

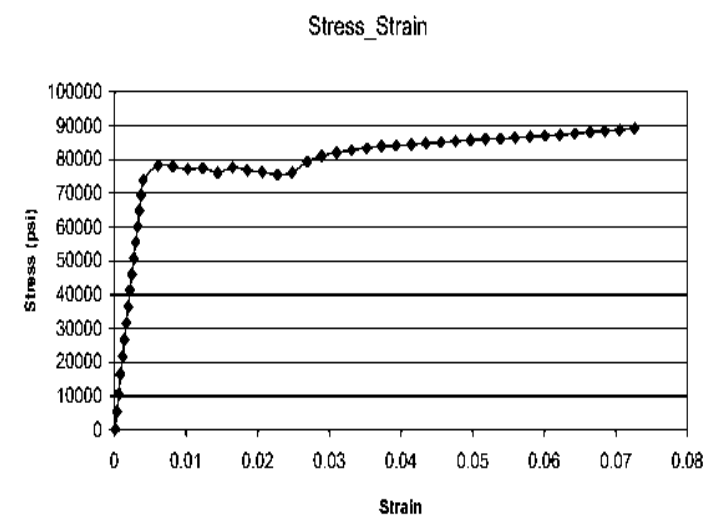

Fig 9: Stress-strain curve for NDC super-elasticity materials' width of 0.187

Fig 10 and 11 indicate the correspondent curve for NDC SMA (SM-495) wire with an approximate diameter of 0.14. In Fig 11 , the readings of plateau of the stress-strain curve are approximately $13 \mathrm{~K}$ psi that is next to $15 \mathrm{~K}$ psi issues by the firm. Young's modulus evaluated from Fig 11 is 8.14 by 106 whereas one issued by the firm is 4.6 by 106 psi.

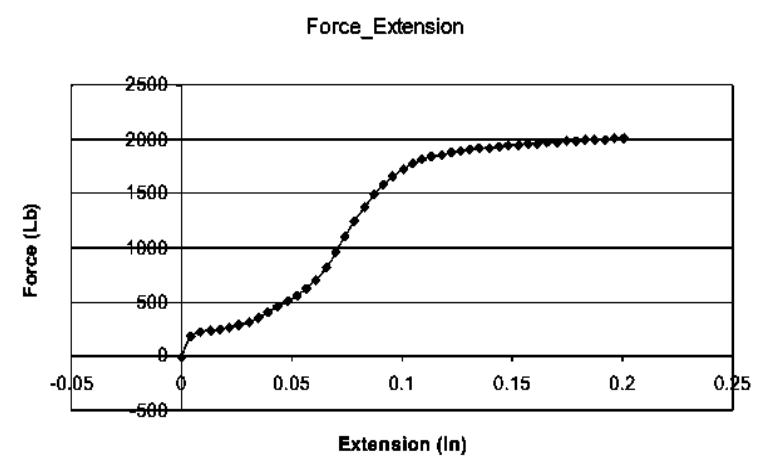

Fig 10: Load versus extension curve for NDC SMs with width of 0.14 
Stress_Strain

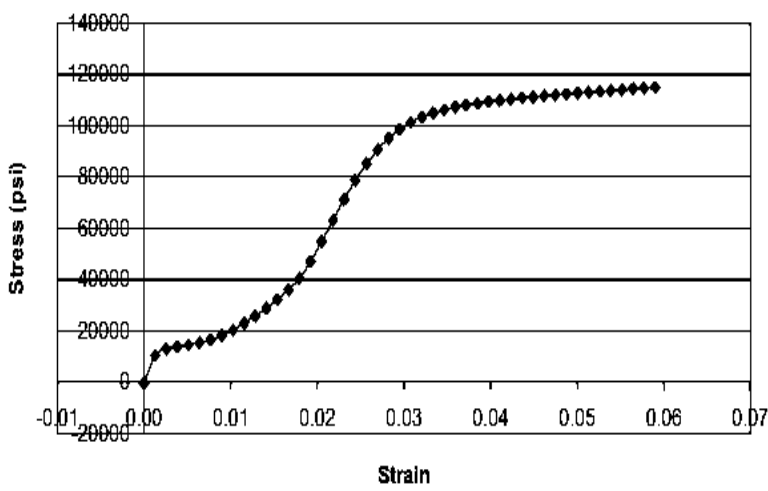

Fig 11: Stress versus strain curve for NDC SMs with width of about 0.41

The strength has been evaluated from the test information and is close to the values given by the firm, whereas Young's modulus is more than the provided value. The major reason is that due to the fact that the sample considered is smaller, the surface is significantly smooth and there is slipperiness between the sample and extensometer and clamp as well. The readings of the force have not in any way been affected; however, there is slipperiness between the sample and clamp whereas the extension readings are less compared to the real values.

Based on the two factors, Young's modulus being calculated must be more than the real one, The curve of stress against strain of super-elasticity material and SMA can be fit for Fig 5 (a). SMs have a post yield timeline with higher strength compared to yield strengths. After NDC SMs have been pulled, it is placed into oven as presented in the methodology section. Due to the fact that SMs have been over-stretched into strengthened period (because of its slipperiness), it is challenging to control the effect of pulling and it might even shift to its initial length through heating.

Before the beam experiments are carried out, the tests of cylinders that are concrete were done. In these tests, the mean strength of the concrete at 30 days was considered 3810.5 psi. From the geometric and material parameters in the methodology section, theoretical cracking and nominal moments were evaluating with reference to Table 5 below, whereby cracking moments have been shown by Mc, Mn has represented Nominal moments and Pn and Pc represent the forces that correspond to $\mathrm{Mn}$ and Mc. The pre-evaluated forces and moments are essential in the process of controlling the process of loading.

Table 5: Mc and Mn of sunbeams

\begin{tabular}{lllll}
\hline & $\begin{array}{l}\text { Sunbeam } \\
\mathbf{1}\end{array}$ & $\begin{array}{l}\text { Sunbeam } \\
\mathbf{2}\end{array}$ & $\begin{array}{l}\text { Sunbeam } \\
\mathbf{3}\end{array}$ & $\begin{array}{l}\text { Sunbeam } \\
\mathbf{3}\end{array}$ \\
\hline $\begin{array}{l}\text { Mc } \\
\text { (lbln) }\end{array}$ & 8662.10 & 8570.70 & 8548.80 & 4342.70 \\
\hline $\begin{array}{l}\text { Mn } \\
\text { (lbln) }\end{array}$ & 11880.80 & 20006.60 & 18108.90 & 10134.90 \\
\hline Pc (lb) & 1443.70 & 1428.50 & 1424.80 & 723.70 \\
\hline Pn (lb) & 2464.30 & 3334.40 & 3018.20 & 1689.10 \\
\hline
\end{tabular}

In the experiments, four beams have been shown in sequence. The experiment was successful except for the controller beam due to improper operations of acquiring data during the control beam experiments, which led to a fail in the process of collecting the experimental information. Out of the four beams, only a single visible crack was evident from every beam during the complete process of loading. This was partly because of the slipperiness between the concrete and the wires that stopped the development of uniform distributed cracking reinforcement concrete beams.

High pitch noises can be heard and the wire slipperiness was due to the wire ends being observed to shift into the concrete. This critical concern may have amounted to critical anomalous findings. Based on the theories of simple beams, cracking has to be at the top surface of the supports since load is applicable upwards and bends moments at the middle span, which is minimal compared to the loading part because of the downward custom-weight effect.

\section{CONCLUSION AND FUTURE DIRECTIONS}

From the discussion of the results in the above section, it is seen that SMs have proved their application in structural engineering and critical analysis has been done in this field of research. The major mechanical element of SMs incorporate 
SM effect and super-elasticity as seen in material testing, mostly in the analysis of stress versus strain curves. The curves relate significantly based on the overall features. Super-elasticity material testing had higher yield so that under normal force, materials will be elastic. In that regard, it is capable of shifting back to its initial shape after stress or force has been released. Above this stress, the mode of recovery is dependent on the staged transformation. From the findings of the beam evaluation, super-elasticity materials can recover the cracking mode of the concrete in some degree after external stress is diminished.

Nonetheless, gravity can be affected and results altered. Therefore, careful experimental models are required. The slipperiness of the wire surface was critical in both pulling and beam tests, and this issue can potentially cause anomalous results in future experiments. Future researchers should focus on applying critical experimental models to avoid the issue of slipperiness of the surface of wires in beam and pull tests to achieve significant results. In addition, this contribution has been added to literature to project the analysis of pre-stress, custom repair capacities and two-path memory training. This contribution has been projected to enhance the development of SMs and smart bridges in future.

\section{References}

[1]. D. Farhey, "Structural Performances of Bridge Materials in the U.S. National Bridge Inventory 2013", Structural Engineering International, vol. 27, no. 1, pp. 101-113, 2017. Available: 10.2749/101686617x14676303589039.

[2]. X. Feng, L. Zhao and X. Mi, "Characristics of Shape Memory Composites Combined with Shape Memory Alloy and Shape Memory Polymer", Advanced Materials Research, vol. 705, pp. 169-172, 2013. Available: 10.4028/www.scientific.net/amr.705.169.

[3]. A. Volkov, M. Evard, A. Vikulenkov and E. Uspenskiy, "Simulation of Vibration Isolation by Shape Memory Alloy Springs Using a Microstructural Model of Shape Memory Alloy", Materials Science Forum, vol. 738-739, pp. 150-154, 2013. Available: 10.4028/www.scientific.net/msf.738-739.150.

[4]. G. LIU, H. PENG, Y. WEN, K. YANG and N. LI, "A TRAINING--FREE CAST Fe--Mn--Si--Cr--Ni SHAPE MEMORY ALLOY BASED ON FORMATION OF MARTENSITE IN A DOMAIN MANNER II. Influence of annealing on shape memory effect", ACTA METALLURGICA SINICA, vol. 46, no. 3, pp. 288-293, 2010. Available: 10.3724/sp.j.1037.2010.00288.

[5]. O. Ostertag and E. Ostertagová, "Shape Memory Alloy Actuator (SMA)", Applied Mechanics and Materials, vol. 816, pp. 9-15, 2015. Available: 10.4028/www.scientific.net/amm.816.9.

[6]. H. Wang, J. Sui, C. Liu and W. Cai, "Martensitic transformation and shape memory effect in Ni54.75Mn13.25Fe7Ga25 ferromagnetic shape memory alloy", Materials Science and Engineering: A, vol. 480, no. 1-2, pp. 472-476, 2008. Available: 10.1016/j.msea.2007.07.053.

[7]. M. Akita, "Photochromic Organometallics, A Stimuli-Responsive System: An Approach to Smart Chemical Systems $\dagger$ ", Organometallics, vol. 30, no. 1, pp. 43-51, 2011. Available: 10.1021/om100959h.

[8]. J. Clark, "Designing the Smart City: A Programmatic Approach to Inclusive Innovation in Atlanta", Atlanta Studies, 2017. Available: 10.18737/atls20170919.

[9]. S. Kim and H. Sohn, "Instantaneous reference-free crack detection based on polarization characteristics of piezoelectric materials", Smart Materials and Structures, vol. 16, no. 6, pp. 2375-2387, 2007. Available: 10.1088/0964-1726/16/6/042.

[10]. W. Choi, "Special Issue: Smart Nanomaterials for Environmental Remediation", Materials, vol. 14, no. 1, p. 143, 2020. Available: $10.3390 /$ ma14010143.

[11]. J. Zhang and C. McInnes, "Reconfiguring smart structures using approximate heteroclinic connections", Smart Materials and Structures, vol. 24, no. 10, p. 105034, 2015. Available: 10.1088/0964-1726/24/10/105034.

[12]. S. Klinkel, D. Legner and W. Wagner, "Advanced finite element formulations for modeling thin piezoelectric structures", PAMM, vol. 11, no. 1, pp. 31-34, 2011. Available: 10.1002/pamm.201110009.

[13]. T. Breczko, V. Barkaline and Y. Douhaya, "Modeling of Smart Material Properties of Heusler Alloys", Materials Science Forum, vol. 738-739, pp. 426-430, 2013. Available: 10.4028/www.scientific.net/msf.738-739.426.

[14]. D. Vojtěch and A. Michalcová, "Influence of Heat-Treatment on Mechanical Properties and Transformation Temperatures of Nitinol", Key Engineering Materials, vol. 465, pp. 471-474, 2011. Available: 10.4028/www.scientific.net/kem.465.471.

[15]. S. Dokhov, "Measurement of the temperatures in the thermostats of laboratory chromatographs", Measurement Techniques, vol. 24, no. 12, pp. 1107-1107, 1981. Available: 10.1007/bf00828732.

[16]. B. Zhou and S. Yoon, "A new phase transformation constitutive model of shape memory alloys", Smart Materials and Structures, vol. 15, no. 6, pp. 1967-1973, 2006. Available: 10.1088/0964-1726/15/6/053.

[17]. H. Wang and Z. Zhu, "Energy-efficient and reference-free monotonic capacitor switching scheme with fewest switches for SAR ADC", IEICE Electronics Express, vol. 12, no. 7, pp. 20141202-20141202, 2015. Available: 10.1587/elex.12.20141202.

[18]. M. Shahmardani, N. Vajragupta and A. Hartmaier, "Robust Optimization Scheme for Inverse Method for Crystal Plasticity Model Parametrization", Materials, vol. 13, no. 3, p. 735, 2020. Available: 10.3390/ma13030735. 